\title{
GMR
}

\section{Effects of ginsenoside Rg1 on glucose metabolism and liver injury in streptozotocin-induced type 2 diabetic rats}

\author{
W. Tian ${ }^{1 *}$, L. Chen ${ }^{1,2 *}$, L. Zhang ${ }^{1}$, B. Wang ${ }^{1}$, X.B. Li ${ }^{1}$, K.R. Fan ${ }^{3}$, C.H. Ai ${ }^{4}$, \\ X. Xia ${ }^{1}$, S.D. $\mathrm{Li}^{5}$ and Y. Li ${ }^{1}$ \\ ${ }^{1}$ Department of Cadre Health, \\ First People's Hospital of Yunnan Province, Kunming, Yunnan, China \\ ${ }^{2}$ Department of Biomedical Science, School of Medicine, \\ Kunming University of Science and Technology, Kunming, Yunnan, China \\ ${ }^{3}$ School of Life Science and Technology, \\ Southwest Jiaotong University, Chongqing, China \\ ${ }^{4}$ Department of Basic Medicine, \\ Yunnan University of Traditional Chinese Medicine, Kunming, Yunnan, China \\ ${ }^{5}$ Department of Basic Medicine, Kunming Medical University, Kunming, \\ Yunnan, China \\ *These authors contributed equally to this study. \\ Corresponding authors: Y. Li / S.D. Li \\ E-mail: liyanken@126.com / shudeli006@vip.sina.com
}

Genet. Mol. Res. 16 (1): gmr16019463

Received October 20, 2016

Accepted February 16, 2017

Published March 30, 2017

DOI http://dx.doi.org/10.4238/gmr16019463

Copyright $(2017$ The Authors. This is an open-access article distributed under the terms of the Creative Commons Attribution ShareAlike (CC BY-SA) 4.0 License.

\begin{abstract}
Type 2 diabetes mellitus (T2-DM) is a chronic metabolic disorder characterized by high blood glucose levels. T2-DM patients suffer from many complications, such as diabetic fatty liver and diabetic nephropathy. The liver, the pivotal organ involved in both glucose and lipid metabolism, is primarily damaged in T2-DM patients, especially in those with high levels of blood lipid. In this study, the hepatoprotective activity of ginsenoside $\mathrm{Rg} 1$ was investigated in a
\end{abstract}

Genetics and Molecular Research 16 (1): gmr16019463 
T2-DM rat model. The results revealed a potent hepatoprotective effect of ginsenoside $\mathrm{Rg} 1$. This effect was primarily mediated by the antiapoptotic effect, inhibition of JNK activity, and suppression of inflammation after ginsenoside $\operatorname{Rg} 1$ treatment. Ginsenoside $\operatorname{Rg} 1$ also lowered the blood glucose level and insulin resistance index in T2-DM rats. Moreover, the blood lipid profile (total cholesterol, triglycerides, and low-density lipoprotein cholesterol levels) and liver function (aspartate transaminase and alanine transaminase levels) improved after ginsenoside $\mathrm{Rg} 1$ treatment. The aforementioned hepatoprotective effects of ginsenoside Rg1 in the T2-DM rat model suggests its clinical potential as an adjuvant drug for T2-DM therapy, especially for T2-DM patients with fatty liver disease.

Key words: Ginsenoside Rg1; Type 2 diabetes mellitus; Hepatoprotection; Apoptosis

\section{INTRODUCTION}

Diabetes mellitus (DM) is an endocrinal and metabolic disease featuring high blood glucose (de Azevêdo Silva et al., 2015; Ghodsian et al., 2015). Following cardiovascular disease and cancer, DM is the third serious chronic, non-infectious disease threatening human health. Currently, there are approximately 230 million patients worldwide with the highest disease prevalence in China (Wong and Wang, 2006; Xu et al., 2013). By 2030, the number of global diabetic patients will approximate 336 million, and the disease will also present at a younger age (Wild et al., 2004). With increasing morbidity and mortality, diabetes and diabetic complications have attracted considerable attention. Furthermore, the majority of patients with diabetes have type 2 diabetes mellitus (T2-DM) (Goldenberg and Punthakee, 2013).

The liver, an important metabolic organ, is also a target organ of insulin. Liver damage can cause an imbalance in glucose and blood lipid levels, and lead to many other metabolic disorders. Studies have also shown that T2-DM can cause liver disease (Lo et al., 2011; Zein et al., 2011; Fukuda et al., 2016). Approximately 21-78\% patients with T2-DM also exhibit fatty liver (McCullough, 2002). The liver is primarily damaged in T2-DM patients, especially in those with high blood lipid levels. However, the mechanism of T2-DM causing liver disease is not yet fully known.

Ginsenoside $\mathrm{Rg} 1$ is one of the most important components of Panax ginseng (Zhang et al., 2012; Yue Z et al., 2014; Ma et al., 2016; Xiao et al., 2016). P. ginseng has been used for the treatment of various diseases for thousands of years (Yu et al., 2015). Studies have shown that ginsenoside Rg1 has anti-inflammatory, antioxidant, immunomodulatory, cardiovascular, and neuronal protective effects (Zhu et al., 2009; Lee and Kim, 2014; Lee et al., 2015; Gao et al., 2015; Sun et al., 2016). Few studies have investigated the effect of ginsenoside Rg1 on liver damage. Thereby, the objective of the present study was to investigate the protective effects of ginsenoside $\operatorname{Rg} 1$ on liver injury in rats with streptozotocin (STZ)- and high-fat diet-induced type 2 diabetes. Furthermore, we examined whether the hepatoprotective effects of ginsenoside Rg1 were mediated through its antiapoptotic effect and inhibition of c-Jun N-terminal kinase (JNK) activity together with its anti-inflammatory effect in the T2-DM rat model.

Genetics and Molecular Research 16 (1): gmr16019463 


\section{MATERIAL AND METHODS}

\section{Materials}

The study protocol was approved by the Animal Care and Use Committee of Kunming Medical University. Male 4-week-old Sprague Dawley rats ( $\mathrm{N}=80,200 \pm 20 \mathrm{~g})$ were purchased from the Animal Center of Kunming Medical University. The rats were housed at $23^{\circ} \pm 2{ }^{\circ} \mathrm{C}$ and $55 \pm 2 \%$ humidity. The animals had free access to tap water and normal rat diet prior to the start of the experiment. Ginsenoside $\operatorname{Rg} 1$ of $>98 \%$ purity measured via high-performance liquid chromatography was obtained from the College of Pharmacy of Kunming Medical University. STZ was obtained from Sigma Chemical Co. (St. Louis, MO, USA). The bicinchoninic acid (BCA) protein assay kit was obtained from TIANGEN Biotech (Beijing) Co., Ltd. Glucose and insulin enzyme-linked immunosorbent assay (ELISA) kits were purchased from BioSwamp Life Science Lab, China. Mouse monoclonal anti-JNK, anti-phospho-JNK (p-JNK), anti-caspase-3, anti-BCL-2-associated X protein (BAX), anti-B cell leukemia/lymphoma 2 (BCL-2), and anti- $\beta$-actin were obtained from Santa Cruz Biotechnology (Santa Cruz, CA). Goat anti-mouse antibody labeled with horseradish peroxidase was obtained from Beijing Zhongshan Golden Bridge Biotechnology Co., Ltd., Beijing, China.

\section{Experimental protocol}

All the rats were acclimatized for 2 weeks before the start of the experiment. Of the 80 rats, 20 rats were randomly selected as normal controls while the remaining 60 rats received a high-fat diet for 8 weeks, followed by intraperitoneal injection of STZ $(30 \mathrm{mg} / \mathrm{kg}$ in $0.1 \%$ citric acid buffer solution, $\mathrm{pH} 4.5$ ) to induce T2-DM. Blood glucose levels were determined with a SureStep ${ }^{\mathrm{TM}}$ Plus blood glucose meter (Johnson \& Johnson Co., New Brunswick, NJ, USA). T2-DM was considered as successfully induced when 3 days after STZ injection, the rat blood glucose levels were $>16.7 \mathrm{mM}$ for three consecutive days. The 60 rats that developed diabetes were randomly divided into three groups as follows: the model control group ( $\mathrm{N}$ $=20)$, the high-dose $\left(50 \mathrm{mg} \cdot \mathrm{kg}^{-1} \cdot \mathrm{day}^{-1}\right)$ ginsenoside $\mathrm{Rg} 1$ treatment group $(\mathrm{N}=20)$, and the low-dose $\left(25 \mathrm{mg} \cdot \mathrm{kg}^{-1} \cdot \mathrm{day}^{-1}\right)$ ginsenoside $\mathrm{Rg} 1$ treatment group $(\mathrm{N}=20)$. Ginsenoside $\mathrm{Rg} 1$ was administered daily via gavage for 8 weeks. Rats in the normal control and model control groups received the same volumes of $0.9 \%$ saline solution.

\section{Sample collection}

After 4 or 8 weeks of treatment, half the rats from each group were weighed and anesthetized with $3.6 \%$ chloral hydrate $(8 \mathrm{~mL} / \mathrm{kg}$, ip), and were affixed to the operating table. Blood samples obtained from the heart were allowed to stand for $30 \mathrm{~min}$, and were then centrifuged at $3850 \mathrm{~g}$ for $15 \mathrm{~min}$ at $4^{\circ} \mathrm{C}$. The supernatant was collected and frozen at $-80^{\circ} \mathrm{C}$ for subsequently determining the levels of blood glucose, interleukin (IL)-1, IL-6, tumor necrosis factor-alpha (TNF- $\alpha)$, total cholesterol (TC), triglycerides (TG), low-density lipoprotein cholesterol (LDL-C), aspartate transaminase (AST), and alanine transaminase (ALT). After blood sample collection, the livers were dissected. The harvested liver tissue was cut into two, and one part was immediately frozen at $-80^{\circ} \mathrm{C}$ until western blot analysis, while the remaining tissue was fixed in $10 \%$ neutral-buffered formalin.

Genetics and Molecular Research 16 (1): gmr16019463 


\section{Hematoxylin and eosin staining}

Isolated liver tissues were embedded in paraffin and cut into 4- $\mu \mathrm{m}$-thick sections for subsequent hematoxylin and eosin (HE) staining as per a standard procedure. The changes and inflammatory cell infiltration in the liver tissues were observed using an Olympus AX70 microscope (Olympus, Tokyo, Japan).

\section{Enzyme-linked immunosorbent assay}

Serum insulin was measured via ELISA following the manufacturer protocol (product number: HM10073).

\section{Determination of inflammatory reaction factors and the parameters of liver function and blood lipids}

Inflammatory reaction factors (IL-1, IL-6, and TNF- $\alpha$ ), blood lipids (TC, TG, and LDL-C), and liver function (AST and ALT) were detected using an AU400 automatic biochemical analyzer (Olympus).

\section{Determination of relevant protein levels}

Western blot analysis was used to detect JNK, p-JNK, caspase-3, BCL-2, and BAX expression levels in the liver tissues. Briefly, total protein from the liver was extracted using the radioimmunoprecipitation assay buffer via a standard procedure, and the protein concentration was determined using the BCA protein assay kit. All the proteins were separated using $10 \%$ SDS-PAGE and transferred onto nitrocellulose membranes. The membranes were blocked with $5 \%$ bovine serum albumin for $60 \mathrm{~min}$, followed by overnight incubation with the primary antibodies against JNK, p-JNK, caspase-3, BCL-2,BAX, and $\beta$-actin (1:500 dilution) at $4^{\circ} \mathrm{C}$. The membranes were then washed three times with Tris-buffered saline-Tween and incubated with the appropriate secondary antibody at a dilution of 1:500 for $120 \mathrm{~min}$. Proteins were detected using an enhanced chemiluminescence reagent, Merck Millipore, Co., Ltd., Beijing, China. The densitometric quantification of the bands was carried out using Image-Pro Plus 6.0 system (Media Cybernetics, Shanghai, China) and expressed relative to $\beta$-actin.

\section{Data analyses}

Data are reported as means $\pm \mathrm{SD}$. Comparisons between study groups were made via one-way analysis of variance, followed by Bonferroni post-hoc test. Data were analyzed using SPSS software (version 19.0; SPSS Inc., Chicago, IL, USA). P $<0.05$ was considered statistically significant. Western blot densitometric values are reported in arbitrary units (A.U.).

\section{RESULTS}

\section{General observations}

The rats in the normal control group had normal body weight and white shiny fur, 
while the rats treated with the high-fat diet and STZ injection had noticeably withered and dry fur. Moreover, polydipsia, polyuria, or polyphagia symptoms gradually worsened with a slower reaction time after STZ injection. Compared with the untreated diabetic rats, those treated with ginsenoside Rg1 had a good physical state mentioned above. The high-dose treated rats were noticeably better in physical conditions than the low-dose treatment group.

\section{Histological evaluation}

Hepatoprotection from lipid-induced and inflammatory damages is important, especially for patients with non-alcoholic steatohepatitis. In this study, via histological evaluation, ginsenoside $\operatorname{Rg} 1$ was found to show a hepatoprotective effect in the T2-DM rat model (Figure 1). Compared with the normal control group (Figure 1A and E), the hepatic tissue of the T2-DM rats was infiltrated by inflammatory cells and the structure of the hepatic lobule was abnormal with increasing volume of liver cells (hydropic degeneration) and fatty change in the model control group (Figure 1B and F). However, these hepatic changes improved after ginsenoside Rg1 treatment for 4 and 8 weeks (Figure 1C, G, D, and H).
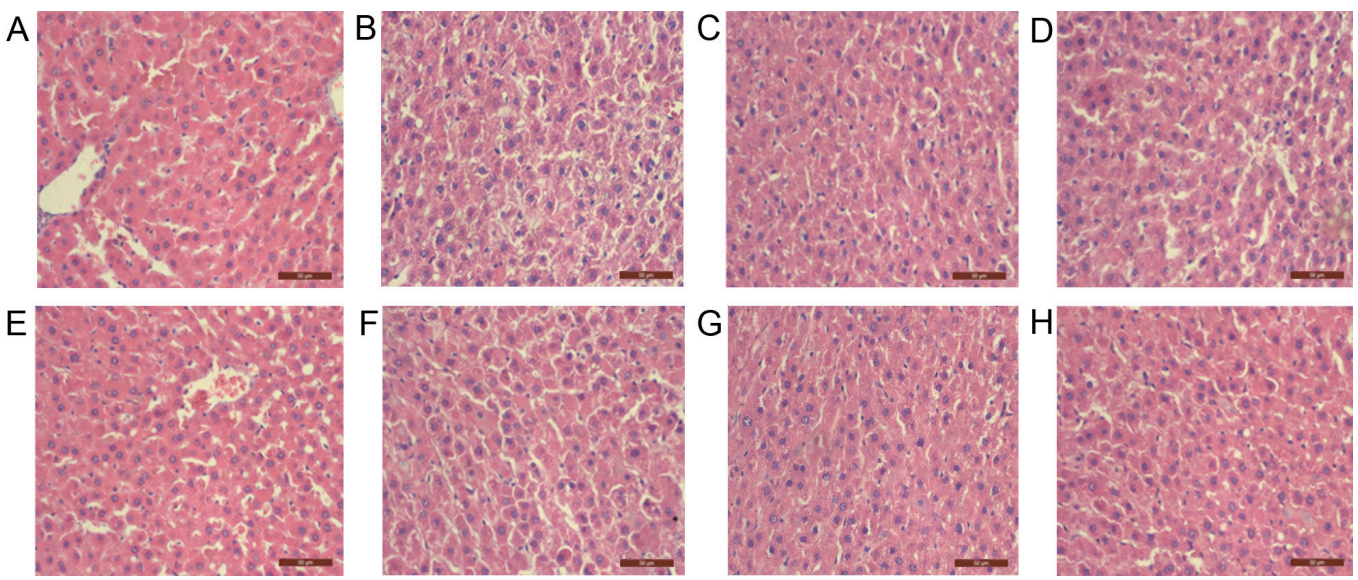

Figure 1. Ginsenoside Rg1 ameliorated pathological liver damage in T2-DM rats. A. B. C. D. H\&E-stained liver sections of normal control group, model control group, low-dose treatment group $\left(25 \mathrm{mg} \cdot \mathrm{kg}^{-1} \cdot \mathrm{day}^{-1}\right)$, and high-dose treatment group ( $50 \mathrm{mg} \cdot \mathrm{kg}^{-1} \cdot \mathrm{day}^{-1}$ ) with $\mathrm{Rg} 1$ for 4 weeks, respectively; while $\mathbf{E}-\mathbf{H}$ correspond to the same groups with or without ginsenoside Rg1 treatment for 8 weeks.

\section{Ginsenoside Rg1 improved glucose metabolism}

DM is primarily characterized by high blood glucose level, and maintaining normal blood glucose level is of clinical significance. To investigate whether ginsenoside Rg1 lowered blood glucose levels and improved its metabolism, the glucose and insulin levels, and insulin resistance index (IRI) were monitored in the T2-DM rats after treatment with ginsenoside Rg1. The results showed that ginsenoside Rg1 potently decreased the blood glucose level and IRI in a dose- and time-dependent manner as compared to that in the model control group (Table 1). Meanwhile, the blood glucose levels and IRI in the model control group were remarkably higher than those of the control group, indicating a successful T2-DM rat model. However, the effect of ginsenoside $\operatorname{Rg} 1$ on insulin level was not noticeable.

Genetics and Molecular Research 16 (1): gmr16019463 
Table 1. Glucose and insulin levels, and IRI in T2-DM rats with different ginsenoside Rg1 treatments.

\begin{tabular}{l|c|c|c|c|c|c}
\hline & \multicolumn{2}{|c|}{ Glu $(\mathrm{mM})$} & \multicolumn{2}{c|}{ Insulin (mU/L) } & \multicolumn{2}{c}{ IRI } \\
\cline { 2 - 7 } & 4 weeks & 8 weeks & 4 weeks & 8 weeks & 4 weeks & 8 weeks \\
\hline Normal control & $6.4 \pm 1.0$ & $6.1 \pm 0.9$ & $10.1 \pm 1.0$ & $10.2 \pm 0.4$ & $2.21 \pm 0.11$ & $2.36 \pm 0.27$ \\
\hline Model control & $24.9 \pm 1.5$ & $27.3 \pm 1.5$ & $12.3 \pm 0.6$ & $12.6 \pm 0.7$ & $13.32 \pm 0.24$ & $15.21 \pm 0.16$ \\
\hline Low-dose treatment & $19.5 \pm 1.0^{*}$ & $17.3 \pm 1.6^{*}$ & $12.9 \pm 0.5$ & $11.6 \pm 1.1$ & $11.18 \pm 0.17^{*}$ & $10.92 \pm 0.09^{*}$ \\
\hline High-dose treatment & $15.6 \pm 1.1^{*}$ & $13.5 \pm 0.9^{*}$ & $11.9 \pm 0.7$ & $10.9 \pm 0.5$ & $8.25 \pm 0.30^{*}$ & $7.54 \pm 0.34^{*}$ \\
\hline
\end{tabular}

$* \mathrm{P}<0.05$, compared with model control. Results are presented as mean $\pm \mathrm{SEM}, \mathrm{N}=10$. Glu, glucose level; IRI, insulin resistance index.

\section{Ginsenoside Rg1 improved hepatic function}

To evaluate the improvement of hepatic function by ginsenoside $\operatorname{Rg} 1$ in T2-DM rat model, AST and ALT levels were detected. As shown in Table 2, compared with the normal control group, the rats in the model control group had significantly higher mean serum levels of AST and ALT. However, with the ginsenoside Rg1 treatment, the levels of AST and ALT decreased substantially in a dose- and time-dependent manner (all $\mathrm{P}<0.05)$.

Table 2. Liver function of T2-DM rats after different ginsenoside $\mathrm{Rg} 1$ treatments.

\begin{tabular}{l|c|c|c|c}
\hline & \multicolumn{2}{|c|}{ AST (U/L) } & \multicolumn{2}{c}{ ALT (U/L) } \\
\cline { 2 - 5 } & 4 weeks & 8 weeks & 4 weeks & 8 weeks \\
\hline Normal control & $66.9 \pm 4.7$ & $69.7 \pm 5.1$ & $47.7 \pm 6.4$ & $49.2 \pm 7.0$ \\
\hline Model control & $148.3 \pm 6.6$ & $150.3 \pm 4.7$ & $133.5 \pm 8.2$ & $134.2 \pm 6.2$ \\
\hline Low-dose treatment & $118.2 \pm 14.4^{*}$ & $107.3 \pm 12.9^{*}$ & $117.5 \pm 5.0^{*}$ & $110.2 \pm 8.1^{*}$ \\
\hline High-dose treatment & $95.9 \pm 4.1^{*}$ & $87.3 \pm 6.6^{*}$ & $94.2 \pm 6.3^{*}$ & $89.1 \pm 6.5^{*}$ \\
\hline
\end{tabular}

$* \mathrm{P}<0.05$, compared with model control. AST, aspartate aminotransferase; ALT, alanine aminotransferase.

\section{Ginsenoside Rg1 ameliorated blood lipid metabolic effects}

The T2-DM rat model used in this study was established using STZ-injection combined with a high-fat diet. Thus, to clarify the effect of ginsenoside Rg1 on blood lipid levels, TC, TG, and LDL-C levels were measured. The results showed that ginsenoside Rg1 effectively reduced TC, TG, and LDL-C serum levels in a dose-dependent manner, while the time course effect was not remarkable (Table 3 ).

Table 3. Blood lipid profile of T2-DM rats after different ginsenoside Rg1 treatments.

\begin{tabular}{l|c|c|c|c|c|c}
\hline & \multicolumn{2}{|c|}{ TC $(\mathrm{mM})$} & \multicolumn{2}{c|}{ TG (mM) } & \multicolumn{2}{c}{ LDL-C (mM) } \\
\cline { 2 - 7 } & 4 weeks & 8 weeks & 4 weeks & 8 weeks & 4 weeks & 8 weeks \\
\hline Normal control & $1.13 \pm 0.10$ & $1.10 \pm 0.09$ & $0.16 \pm 0.03$ & $0.15 \pm 0.06$ & $0.13 \pm 0.04$ & $0.12 \pm 0.06$ \\
\hline Model control & $1.38 \pm 0.18$ & $1.37 \pm 0.16$ & $0.40 \pm 0.13$ & $0.45 \pm 0.02$ & $0.26 \pm 0.08$ & $0.27 \pm 0.09$ \\
\hline Low-dose treatment & $1.29 \pm 0.12^{*}$ & $1.26 \pm 0.11^{*}$ & $0.32 \pm 0.01^{*}$ & $0.36 \pm 0.17^{*}$ & $0.18 \pm 0.03^{*}$ & $0.22 \pm 0.06^{*}$ \\
\hline High-dose treatment & $1.19 \pm 0.09^{*}$ & $1.14 \pm 0.12^{*}$ & $0.23 \pm 0.05^{*}$ & $0.20 \pm 0.07^{*}$ & $0.15 \pm 0.03^{*}$ & $0.14 \pm 0.05^{*}$ \\
\hline
\end{tabular}

$* \mathrm{P}<0.05$, compared with model control. TC, total cholesterol; TG, triglyceride; LDL-C, low-density lipoprotein cholesterol.

\section{Ginsenoside Rg1 reduced inflammation by inhibiting JNK activity}

DM patients often have chronic inflammation. Therefore, to validate the antiinflammatory effect of ginsenoside Rg1 in the T2-DM rat model, the levels of inflammatory

Genetics and Molecular Research 16 (1): gmr16019463 
factors IL-1, IL-6, and TNF- $\alpha$ were examined. As shown in Table 4, the model control group had significantly higher mean serum levels of IL-1, IL-6, and TNF- $\alpha$ than the rats in normal control group did. However, after 4 or 8 weeks of treatment with ginsenoside Rg1, the levels of IL-1, IL-6, and TNF- $\alpha$ decreased remarkably in a dose- and time-dependent manner. Furthermore, p-JNK and total-JNK protein levels were detected via western blotting as JNK mediates inflammatory and apoptotic processes. Ginsenoside $\mathrm{Rg} 1$ potently inhibited JNK activity as indicated by the p-JNK level in ginsenoside $\mathrm{Rg} 1$ treatment groups after both 4 and 8 weeks (Figure 2A and B), but had no effect on total-JNK levels (Figure 2A and B). Moreover, JNK transcription level was examined, and the results revealed no significant difference between the treatment and control groups (Figure 2C).

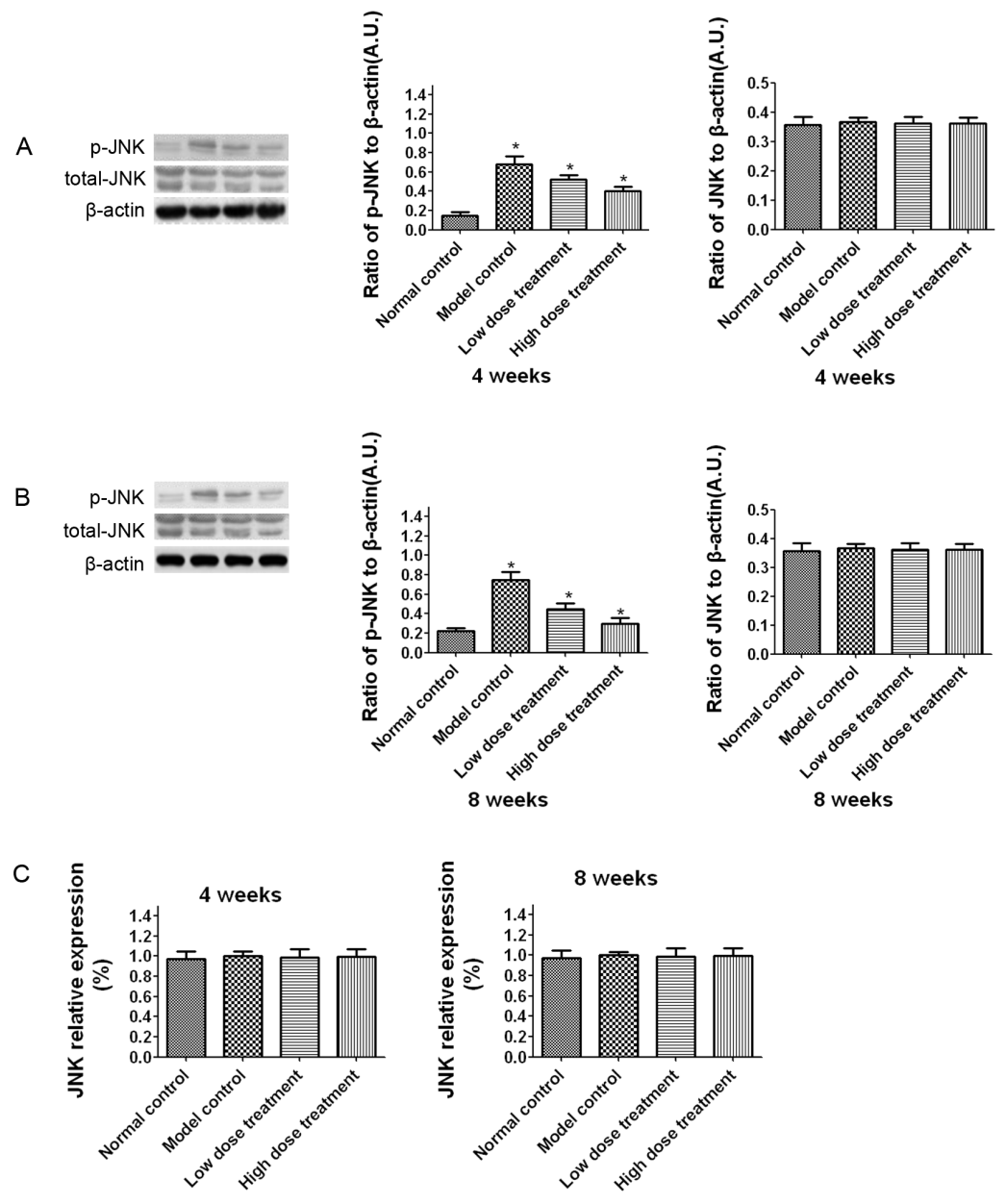

Figure 2. Ginsenoside Rg1 inhibited JNK activity. Ginsenoside Rg1 substantially increased levels of phosphorylated JNK in model control group (T2-DM rat model) as compared to normal control group in 4 (A) and/or 8 weeks (B). However, phosphorylated JNK was reduced after ginsenoside Rg1 treatment for 4 (A) and/or 8 weeks (B) in a dose-dependent manner in both low-dose treatment $\left(25 \mathrm{mg} \cdot \mathrm{kg}^{-1} \cdot\right.$ day $\left.{ }^{-1}\right)$ and high-dose treatment $\left(50 \mathrm{mg} \cdot \mathrm{kg}^{-1} \cdot \mathrm{day}^{-1}\right)$ groups. No significant differences in JNK transcription levels were found among the four groups $(\mathbf{C})$. 
Table 4. Levels of inflammatory cytokines in T2-DM rats after different ginsenoside Rg1 treatments.

\begin{tabular}{l|c|c|c|c|c|c}
\hline & \multicolumn{2}{|c|}{ IL-6 $(\mathrm{pg} / \mathrm{mL})$} & \multicolumn{2}{c|}{ IL-1 (pg/mL) } & \multicolumn{2}{c}{ TNF- $\alpha(\mathrm{pg} / \mathrm{mL})$} \\
\cline { 2 - 7 } & 4 weeks & 8 weeks & 4 weeks & 8 weeks & 4 weeks & 8 weeks \\
\hline Normal control & $64.3 \pm 2.1$ & $62.6 \pm 4.8$ & $88.4 \pm 5.7$ & $89.7 \pm 4.2$ & $36.7 \pm 3.8$ & $36.5 \pm 5.0$ \\
\hline Model control & $138.2 \pm 7.1$ & $137.1 \pm 8.6$ & $175.1 \pm 13.6$ & $180.0 \pm 15.1$ & $118.0 \pm 11.4$ & $120.5 \pm 11.4$ \\
\hline Low-dose treatment & $111.2 \pm 6.2^{*}$ & $99.6 \pm 9.1^{*}$ & $137.4 \pm 5.0^{*}$ & $125.0 \pm 6.7^{*}$ & $91.0 \pm 3.5^{*}$ & $76 \pm 7.2^{*}$ \\
\hline High-dose treatment & $89.3 \pm 4.9^{*}$ & $72.5 \pm 7.2^{*}$ & $100.3 \pm 4.7^{*}$ & $90.4 \pm 5.4^{*}$ & $58.4 \pm 8.5^{*}$ & $48.8 \pm 9.1^{*}$ \\
\hline
\end{tabular}

$* \mathrm{P}<0.05$, compared with model control. Results are presented as mean $\pm \mathrm{SEM}, \mathrm{N}=10$.

\section{Ginsenoside Rg1 inhibited hepatocellular apoptosis}

The liver is often injured as a result of its critical function in glucose and lipid metabolism and detoxification. In patients with diabetes, hepatic damage from elevated blood glucose and lipid levels is common. Hence, to confirm the antiapoptotic effect of ginsenoside Rg1 in the T2-DM rat model, active caspase-3, BCL-2, and BAX protein levels in liver tissue were detected by western blot analysis after ginsenoside $\operatorname{Rg} 1$ treatment. The levels of proapoptotic proteins, active caspase-3 (Figure 3A) and BAX (Figure 3B and C), increased, whereas the level of antiapoptotic protein BCL-2 decreased (Figure 3B and C), in the model control group. However, in ginsenoside Rg1 treatment groups, active caspase-3 (Figure 3A) and BAX (Figure 3B and C) significantly reduced, while BCL-2 (Figure 3B and C) increased in a dose- and time-dependent manner (all $\mathrm{P}<0.05)$.

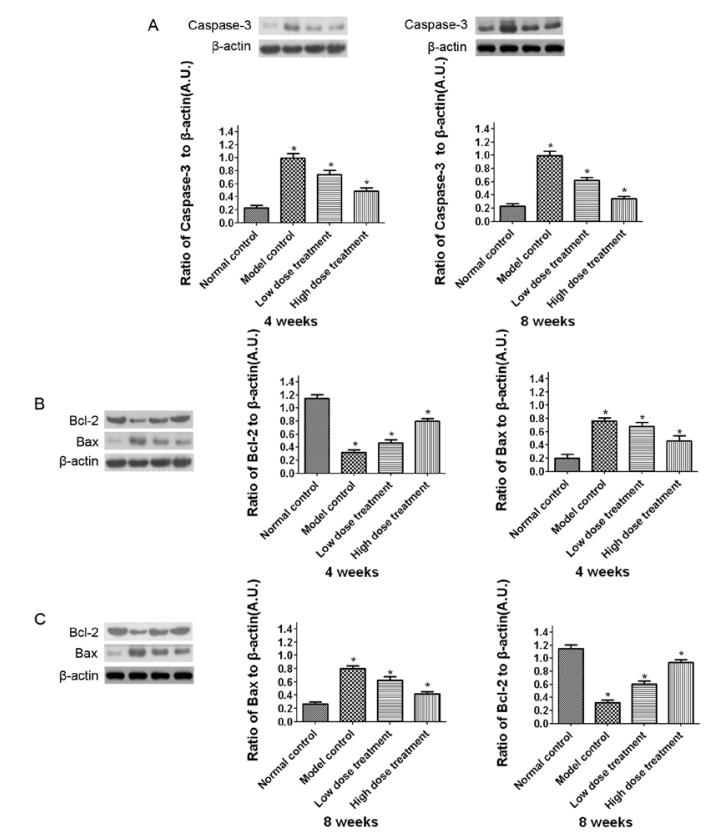

Figure 3. Effect of ginsenoside $\operatorname{Rg} 1$ on apoptotic proteins. Active caspase-3 significantly increased in the model control group (T2-DM rat model) as compared to that in the normal control group after ginsenoside Rg1 treatment for 4 and/or 8 weeks. However, active caspase- 3 decreased potently in low-dose treatment $\left(25 \mathrm{mg} \cdot \mathrm{kg}^{-1} \cdot \mathrm{day}^{-1}\right)$ and high-dose treatment groups (50 mg $\mathrm{kg}^{-1} \cdot \mathrm{day}^{-1}$ ) after 4 and/or 8 weeks (A). Decreased BCL-2 and increased BAX levels were found in model control group compared to normal control group after 4 and 8 weeks $(\mathbf{B}$ and $\mathbf{C})$. Ginsenoside Rg1 treatment restored BCL2 to normal levels in low-dose treatment and high-dose treatment groups after 4 and/or 8 weeks. However, BAX levels decreased substantially in both low-dose treatment and high-dose treatment groups after 4 and/or 8 weeks (B and $\mathbf{C})$. 
Furthermore, the transcription levels of caspase-3, Bax, and $B c l-2$ were monitored. Interestingly, transcription levels of caspase-3 (Figure 4A), Bax (Figure 4C), and Bcl-2 (Figure 4B) demonstrated similar change patterns as that of the proteins between ginsenoside $\operatorname{Rg} 1$ treatment groups and model control group.

A

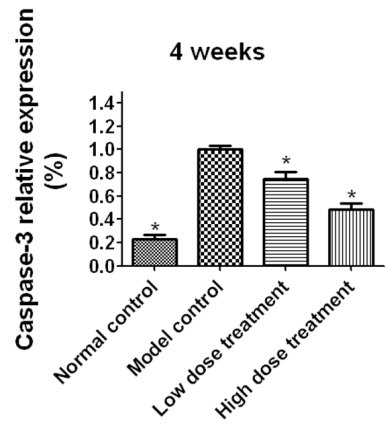

B

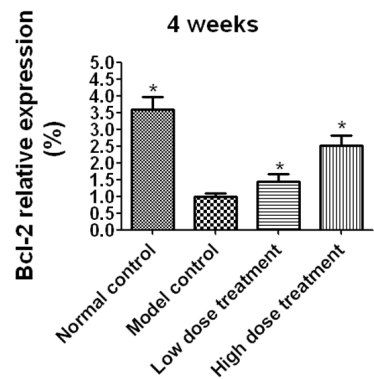

C

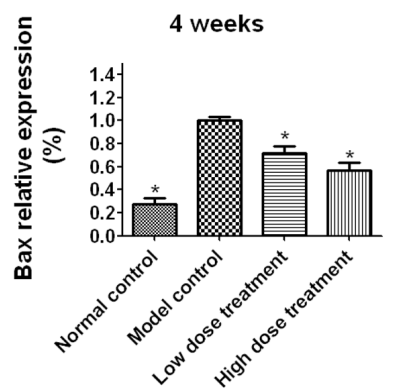

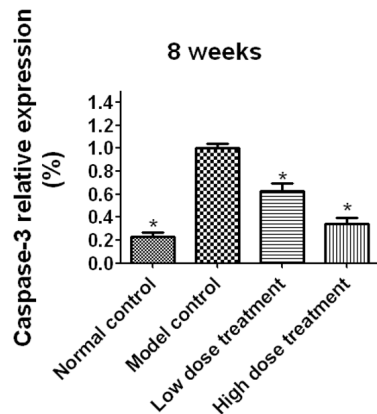
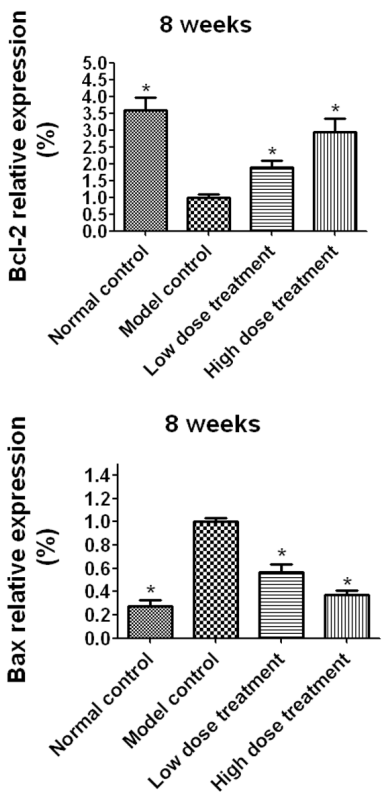

Figure 4. Effect of ginsenoside $\operatorname{Rg} 1$ on transcription levels of apoptotic proteins. Interestingly, the transcription levels of caspase-3 significantly increased in the model control group after 4 and/or 8 weeks compared to that in the normal control group. However, after treatment with ginsenoside $\mathrm{Rg} 1$, caspase-3 levels decreased substantially in a dose-dependent manner in low-dose treatment $\left(25 \mathrm{mg} \cdot \mathrm{kg}^{-1} \cdot \mathrm{day}^{-1}\right)$ and high-dose treatment $\left(50 \mathrm{mg} \cdot \mathrm{kg}^{-1} \cdot \mathrm{day}^{-1}\right)$ groups after 4 and/or 8 weeks (A). Downregulation of Bcl-2 transcription level (B) and upregulation of $B a x$ transcription level (C) were observed in the model control group after 4 and/or 8 weeks. However, elevated $B c l-2$ and decreased Bax transcription levels were observed after ginsenoside Rg1 treatment in low-dose treatment and high-dose treatment groups after 4 and/or 8 weeks in a dose-dependent manner.

\section{DISCUSSION}

In this study, a T2-DM rat model was successfully established with high-fat diet and STZ injection (Skovsø et al., 2015; Chen et al., 2016). Thereafter, the mechanisms underlying 
the hepatoprotective effect of ginsenoside Rg1 in the T2-DM rat were investigated. First, histological evaluation of HE-stained liver sections from the T2-DM rat model showed hepatic tissue infiltration by inflammatory cells, abnormal hepatic lobule structure, and some features of apoptosis. However, the morphology of the hepatic tissue significantly improved after ginsenoside Rg1 treatment. Similarly, liver function, monitored by assessing AST and ALT levels, improved as well. Second, T2-DM-associated blood glucose level and IRI significantly ameliorated after ginsenoside $\mathrm{Rg} 1$ treatment. T2-DM is a chronic disorder characterized by hyperglycemia in the context of insulin resistance, and maintaining normal blood glucose levels has clinical significance. The blood glucose-lowering effect of ginsenoside Rg1 could have clinical therapeutic potential together with first-line anti-diabetic therapeutics. Third, levels of blood lipids including TC, TG, and LDL-C potently reduced after treatment with ginsenoside Rg1. T2-DM patients are usually diagnosed with high levels of blood lipids and have a prevalence of associated non-alcoholic fatty liver disease. In T2-DM patients, high levels of blood lipids cause increased hepatic damage and cardiovascular complications, this aggravates other diabetic complications, such as diabetic nephropathy and diabetic retinopathy. The reduction in blood lipid levels by ginsenoside Rg1 treatment suggests its clinical potential at least for adjuvant therapy. Additionally, inflammation, monitored by measuring the levels of inflammatory cytokines, including TNF- $\alpha$, IL-1, and IL-6, substantially decreased after ginsenoside Rg1 treatment in T2-DM rat model. Meanwhile, p-JNK activity was inhibited as well. Many studies have reported that the inflammation reaction is primarily mediated by the JNK signaling pathway or TNF- $\alpha$-nuclear factor-kappa B (NF- $\kappa B$ ) pathway (Kaminska, 2009; Reber et al., 2009; Zha et al., 2014; Pan et al., 2015; Liu et al., 2016). Whe ther the anti-inflammatory activity of ginsenoside Rg1 in the T2-DM rat model was mediated by the NF- $\mathrm{KB}$ signaling pathway is unknown, and will be further elucidated in our subsequent study. However, the hepatoprotective activity of ginsenoside Rg1 in the T2-DM rat model was attributed to its anti-inflammatory activity. The anti-inflammatory effect of ginsenoside $\operatorname{Rg} 1$ could have therapeutic potential for treating diabetic complications such as diabetic hepatic disease and diabetic renal disease, which are primarily attributed to an imbalance between inflammatory and anti-inflammatory processes.

The underlying mechanism of liver injury in STZ and high-fat diet-induced type 2 diabetic rats is complex. High glucose and inflammatory cytokines can contribute to liver injury. However, the specific mechanism is unclear. Apoptosis of liver cells is an important cause of liver injury. There are two key apoptosis signaling pathways, the mitochondrial and death signal pathways. In the mitochondrial pathway, members of the Bcl-2 family have proapoptotic or antiapoptotic effects (Strasser et al., 2000; Friedlander, 2003; Chen et al., 2012). The proapoptotic/antiapoptotic balance of the Bcl-2 family members, such as Bax (Hengartner and Horvitz, 1994), plays a dominant role in cell apoptosis. Death signal pathway involves first apoptosis signal (Fas) or TNF-RI activated Fas-associated death domain protein (FADD) and caspase-8. Each of the two pathways converges to a common execution phase of apoptosis that requires proteolytic activation of caspase-3 through both the extrinsic and intrinsic signaling pathway (Salvesen and Dixit, 1997; Yamada et al., 1999; Ghavami et al., 2009). Bcl-2, Bax, and active caspase- 3 were three important target molecules in this study. JNK signaling pathway is an important branch of mitogen-activated protein kinase (MAPK) pathways, and activation of the JNK pathway in the long term can lead to cell apoptosis.

A previous study suggested that ginsenoside $\mathrm{Rg} 1$ reduced inflammatory reaction factors in renal tissues and improved renal pathological changes in rats with diabetic nephropathy

Genetics and Molecular Research 16 (1): gmr16019463 
(Ma et al., 2010). Moreover, ginsenoside Rg1 also ameliorated oxidative stress and myocardial apoptosis in STZ-induced diabetic rats (Yu et al., 2015). However, the effect of ginsenoside Rg1 on liver injury has been seldom reported. This study demonstrated that ginsenoside $\operatorname{Rg} 1$ inhibited inflammatory reaction and the activation of JNK signaling pathway to reduce liver cell apoptosis and improve liver injury. However, the apoptotic pathway and specific molecules involved in its mode of action are not completely known. Moreover, the role of MAPK signaling pathway needs to be further explored. Previous studies (Ma et al., 2010; Yu et al., 2015; Yu et al., 2016) have mainly focused on the protective effects of ginsenoside $\operatorname{Rg} 1$ in the diabetic rat model and not in patients with diabetes, which is also a limitation of this study. In a future study, we will evaluate the hepatoprotective effect of ginsenoside $\operatorname{Rg} 1$ in patients with diabetes, especially those with high levels of blood lipids or hepatic adipose disease, which would support its clinical application for T2-DM treatment, at least in adjuvant therapy.

\section{CONCLUSIONS}

In this study, ginsenoside $\operatorname{Rg} 1$ was found to have a hepatoprotective effect in T2DM rats. This hepatoprotection by ginsenoside Rg1 was mainly mediated by its antiapoptotic effect and inhibition of JNK pathway, together with its anti-inflammatory action. Meanwhile, ginsenoside $\operatorname{Rg} 1$ improved blood glucose metabolism, as indicated by the decreased glucose level and IRI after ginsenoside Rg1 treatment. Moreover, the blood lipid levels and hepatic function of the T2-DM rat model were ameliorated after ginsenoside Rg1 treatment. The aforementioned effects converged to provide hepatoprotection in the T2-DM model, which indicates the potential clinical application of ginsenoside Rg1 as an adjuvant drug for T2-DM therapy, especially for T2-DM patients with fatty liver disease.

\section{Conflicts of interest}

The authors declare no conflict of interest.

\section{ACKNOWLEDGMENTS}

Research supported by Joint Special Fund of Kunming Medical University, Science and Technology Office of Yunnan Province, Fund of Applied Basic Research of Yunnan Province, Fund of Longde Wang Academician Workstation of The First Affiliated Hospital of Yunnan Province, and Senile Disease Prevention and Control Research Center of Yunnan Province. The funders had no role in study design, data collection and analysis, decision to publish, or preparation of the manuscript.

\section{REFERENCES}

Chen F, Chen Y, Kang X, Zhou Z, et al. (2012). Anti-apoptotic function and mechanism of ginseng saponins in Rattus pancreatic b-cells. Biol. Pharm. Bull. 35: 1568-1573 http://dx.doi.org/10.1248/bpb.b12-00461

Chen YF, Shibu MA, Fan MJ, Chen MC, et al. (2016). Purple rice anthocyanin extract protects cardiac function in STZinduced diabetes rat hearts by inhibiting cardiac hypertrophy and fibrosis. J. Nutr. Biochem. 31: 98-105 http://dx.doi. org/10.1016/j.jnutbio.2015.12.020

de Azevêdo Silva J, Tavares NA, Santos MM, Moura R, et al. (2015). Meta-analysis of STAT4 and IFIH1 polymorphisms in type 1 diabetes mellitus patients with autoimmune polyglandular syndrome type III. Genet. Mol. Res. 14: 17730-

Genetics and Molecular Research 16 (1): gmr16019463 
17738 http://dx.doi.org/10.4238/2015.December.21.46

Friedlander RM (2003). Apoptosis and caspases in neurodegenerative diseases. N. Engl. J. Med. 348: 1365-1375 http:// dx.doi.org/10.1056/NEJMra022366

Fukuda T, Hamaguchi M, Kojima T, Hashimoto Y, et al. (2016). The impact of non-alcoholic fatty liver disease on incident type 2 diabetes mellitus in non-overweight individuals. Liver Int. 36: 275-283 http://dx.doi.org/10.1111/liv.12912

Gao Y, Chu S, Li J, Li J, et al. (2015). Anti-inflammatory function of ginsenoside Rg1 on alcoholic hepatitis through glucocorticoid receptor related nuclear factor-kappa B pathway. J. Ethnopharmacol. 173: 231-240 http://dx.doi. org/10.1016/j.jep.2015.07.020

Ghavami S, Hashemi M, Ande SR, Yeganeh B, et al. (2009). Apoptosis and cancer: mutations within caspase genes. $J$. Med. Genet. 46: 497-510 http://dx.doi.org/10.1136/jmg.2009.066944

Ghodsian N, Akhlaghi M, Ramachandran V, Heidari F, et al. (2015). Association of TNF-a G308A gene polymorphism in essential hypertensive patients without type 2 diabetes mellitus. Genet. Mol. Res. 14: 18974-18979 http://dx.doi. org/10.4238/2015.December.29.4

Goldenberg R and Punthakee Z; Canadian Diabetes Association Clinical Practice Guidelines Expert Committee (2013). Definition, classification and diagnosis of diabetes, prediabetes and metabolic syndrome. Can. J. Diabetes 37 (Suppl 1): S8-S11 http://dx.doi.org/10.1016/j.jcjd.2013.01.011

Hengartner MO and Horvitz HR (1994). C. elegans cell survival gene ced-9 encodes a functional homolog of the mammalian proto-oncogene bcl-2. Cell 76: 665-676 http://dx.doi.org/10.1016/0092-8674(94)90506-1

Kaminska B (2009). Molecular characterization of inflammation-induced JNK/c-Jun signaling pathway in connection with tumorigenesis. Methods Mol. Biol. 512: 249-264 http://dx.doi.org/10.1007/978-1-60327-530-9 13

Lee $\mathrm{CH}$ and $\mathrm{Kim} \mathrm{JH}$ (2014). A review on the medicinal potentials of ginseng and ginsenosides on cardiovascular diseases. J. Ginseng Res. 38: 161-166 http://dx.doi.org/10.1016/j.jgr.2014.03.001

Lee SY, Jeong JJ, Eun SH and Kim DH (2015). Anti-inflammatory effects of ginsenoside Rg1 and its metabolites ginsenoside Rh1 and 20(S)-protopanaxatriol in mice with TNBS-induced colitis. Eur. J. Pharmacol. 762: 333-343 http://dx.doi.org/10.1016/j.ejphar.2015.06.011

Liu L, Song H, Duan H, Chai J, et al. (2016). TSG-6 secreted by human umbilical cord-MSCs attenuates severe burninduced excessive inflammation via inhibiting activations of P38 and JNK signaling. Sci. Rep. 6: 30121 http://dx.doi. org/10.1038/srep30121

Lo L, McLennan SV, Williams PF, Bonner J, et al. (2011). Diabetes is a progression factor for hepatic fibrosis in a high fat fed mouse obesity model of non-alcoholic steatohepatitis. J. Hepatol. 55: 435-444 http://dx.doi.org/10.1016/j. ihep.2010.10.039

Ma R, Sun L, Chen X, Mei B, et al. (2016). Proteomic Analyses Provide Novel Insights into Plant Growth and Ginsenoside Biosynthesis in Forest Cultivated Panax ginseng (F. Ginseng). Front. Plant Sci. 7: 1 http://dx.doi.org/10.3389/ fpls.2016.00001

Ma X, Xie X, Zuo C and Fan J (2010). [Effects of ginsenoside Rg1 on streptozocin-induced diabetic nephropathy in rats]. Sheng Wu Yi Xue Gong Cheng Xue Za Zhi 27: 342-347

McCullough AJ (2002). Update on nonalcoholic fatty liver disease. J. Clin. Gastroenterol. 34: 255-262 http://dx.doi. org/10.1097/00004836-200203000-00013

Pan H, Chen J, Shen K, Wang X, et al. (2015). Mitochondrial modulation by Epigallocatechin 3-Gallate ameliorates cisplatin induced renal injury through decreasing oxidative/nitrative stress, inflammation and NF-kB in mice. PLoS One 10: e0124775 http://dx.doi.org/10.1371/journal.pone.0124775

Reber L, Vermeulen L, Haegeman G and Frossard N (2009). Ser276 phosphorylation of NF-kB p65 by MSK1 controls SCF expression in inflammation. PLoS One 4: e4393 http://dx.doi.org/10.1371/journal.pone.0004393

Salvesen GS and Dixit VM (1997). Caspases: intracellular signaling by proteolysis. Cell 91: 443-446 http://dx.doi. org/10.1016/S0092-8674(00)80430-4

Skovsø S, Damgaard J, Fels JJ, Olsen GS, et al. (2015). Effects of insulin therapy on weight gain and fat distribution in the HF/HS-STZ rat model of type 2 diabetes. Int. J. Obes. 39: 1531-1538

Strasser A, O'Connor L and Dixit VM (2000). Apoptosis signaling. Annu. Rev. Biochem. 69: 217-245 http://dx.doi. org/10.1146/annurev.biochem.69.1.217

Sun XC, Ren XF, Chen L, Gao XQ, et al. (2016). Glucocorticoid receptor is involved in the neuroprotective effect of ginsenoside Rg1 against inflammation-induced dopaminergic neuronal degeneration in substantia nigra. J. Steroid Biochem. Mol. Biol. 155 (Pt A): 94-103 http://dx.doi.org/10.1016/j.jsbmb.2015.09.040

Wild S, Roglic G, Green A, Sicree R, et al. (2004). Global prevalence of diabetes: estimates for the year 2000 and projections for 2030. Diabetes Care 27: 1047-1053 http://dx.doi.org/10.2337/diacare.27.5.1047

Wong KC and Wang Z (2006). Prevalence of type 2 diabetes mellitus of Chinese populations in Mainland China, Hong

Genetics and Molecular Research 16 (1): gmr16019463 
Kong, and Taiwan. Diabetes Res. Clin. Pract. 73: 126-134 http://dx.doi.org/10.1016/j.diabres.2006.01.007

Xiao J, Chen D, Lin XX, Peng SF, et al. (2016). Screening of Drug Metabolizing Enzymes for the Ginsenoside Compound K In Vitro: An Efficient Anti-Cancer Substance Originating from Panax Ginseng. PLoS One 11: e0147183 http:// dx.doi.org/10.1371/journal.pone. 0147183

Xu Y, Wang L, He J, Bi Y, et al.; 2010 China Noncommunicable Disease Surveillance Group (2013). Prevalence and control of diabetes in Chinese adults. JAMA 310: 948-959 http://dx.doi.org/10.1001/jama.2013.168118

Yamada K, Ichikawa F, Ishiyama-Shigemoto S, Yuan X, et al. (1999). Essential role of caspase-3 in apoptosis of mouse beta-cells transfected with human Fas. Diabetes 48: 478-483 http://dx.doi.org/10.2337/diabetes.48.3.478

Yu H, Zhen J, Yang Y, Gu J, et al. (2016). Ginsenoside Rg1 ameliorates diabetic cardiomyopathy by inhibiting endoplasmic reticulum stress-induced apoptosis in a streptozotocin-induced diabetes rat model. J. Cell. Mol. Med. 20: 623-631 http://dx.doi.org/10.1111/jcmm.12739

Yu HT, Zhen J, Pang B, Gu JN, et al. (2015). Ginsenoside Rg1 ameliorates oxidative stress and myocardial apoptosis in streptozotocin-induced diabetic rats. J. Zhejiang Univ. Sci. B 16: 344-354 http://dx.doi.org/10.1631/jzus.B1400204

Yue Z, Rong J, Ping W, Bing Y, et al. (2014). Gene expression of the p16(INK4a)-Rb and p19(Arf)-p53-p21(Cip/Waf1) signaling pathways in the regulation of hematopoietic stem cell aging by ginsenoside Rg1. Genet. Mol. Res. 13: 10086-10096 http://dx.doi.org/10.4238/2014.December.4.3

Zein CO, Unalp A, Colvin R, Liu YC, et al.; Nonalcoholic Steatohepatitis Clinical Research Network (2011). Smoking and severity of hepatic fibrosis in nonalcoholic fatty liver disease. J. Hepatol. 54: 753-759 http://dx.doi.org/10.1016/j. ihep.2010.07.040

Zha L, Chen J, Sun S, Mao L, et al. (2014). Soyasaponins can blunt inflammation by inhibiting the reactive oxygen species-mediated activation of PI3K/Akt/NF-kB pathway. PLoS One 9: e107655 http://dx.doi.org/10.1371/journal. pone. 0107655

Zhang ZL, Fan Y and Liu ML (2012). Ginsenoside Rg1 inhibits autophagy in H9c2 cardiomyocytes exposed to hypoxia/ reoxygenation. Mol. Cell. Biochem. 365: 243-250 http://dx.doi.org/10.1007/s11010-012-1265-3

Zhu D, Wu L, Li CR, Wang XW, et al. (2009). Ginsenoside Rg1 protects rat cardiomyocyte from hypoxia/reoxygenation oxidative injury via antioxidant and intracellular calcium homeostasis. J. Cell. Biochem. 108: 117-124 http://dx.doi. org/10.1002/jcb. 22233

Genetics and Molecular Research 16 (1): gmr16019463 